\title{
The interaction of laser radiation with tissue in the aspect of generating the process of decellularization in the preparation of animal origin autologous tissue
}

\author{
MAGDALENA KOPERNIK ${ }^{1 *}$, ROMAN MAJOR ${ }^{2}$, GRZEGORZ LIS ${ }^{3}$, PIOTR WILCZEK $^{4,5}$, MACIEJ LIS $^{3}$, \\ GABRIELA IMBIR ${ }^{2}$, ABIR CHROUDA $^{2}$, ALDONA MZYK ${ }^{2}$, ROMAN OSTROWSKI ${ }^{6}$, MAREK SANAK ${ }^{7}$ \\ ${ }^{1}$ AGH University of Science and Technology, Kraków, Poland. \\ ${ }^{2}$ Institute of Metallurgy and Materials Science, Polish Academy of Sciences, Kraków, Poland. \\ ${ }^{3}$ Jagiellonian University Medical College, Department of Histology, Kraków, Poland. \\ ${ }^{4}$ Foundation for Cardiac Surgery Development, Bioengineering Laboratory, Zabrze, Poland. \\ ${ }^{5}$ The President Stanislaw Wojciechowski State University of Applied Sciences in Kalisz, \\ Faculty of Health Sciences, Kalisz, Poland. \\ ${ }^{6}$ Military University of Technology, Institute of Optoelectronics, Warsaw, Poland. \\ ${ }^{7}$ Jagiellonian University Medical College, Department of Medicine, Kraków, Poland.
}

\begin{abstract}
Purpose: The aim of the work was to create an appropriate substrate for organ transplantation using bioactive tissue-based scaffold populated by cells of the graft recipient. The purpose of the modeling was to investigate the mechanical effects of wave loading of aortic and pulmonary tissue material. Methods: The biological properties of tissues of aortic and pulmonary valves were modified by the process of decellularization. The host cells were removed by various physical methods with focus on minimal degradation of the extracellular matrix. Thus, the decellularization process was controlled by histological methods. The tissue decellularization process was simulated by finite element modelling. Results: The mechanical results represented by a displacement at the center of the sample were coherent and the heterogeneity of the distribution of the caves on the surface of the samples was confirmed, both by experiment and in the simulation by the alternate occurrence of local minima and maxima. The latter results from the uneven removal of cells from the effect of the wave causing decellularization were also predicted by the numerical model. Laser radiation had a destructive effect on the components of the extracellular matrix (e.g., collagen and elastic fibers), mainly depending on the fluence and number of pulses in a single exposure. Conclusions: The differences between the valve tissue materials were shown, and the impact of the process of decellularization on the properties of the tissues was analyzed. It should be emphasized that due to low absorption and high scattering, laser radiation can deeply penetrate the tissue, which allows for effective decellularization process in the entire volume of irradiated tissue.
\end{abstract}

Key words: decellularized tissue, aortic valve, finite element modeling, histology, pulmonary valve, laser radiation

\section{Introduction}

Currently available prosthetic heart valves are successfully used clinically, but they have many limitations. Degenerative processes and calcification have a great effect on the durability of biological prostheses [9], [1]. Despite the fact that mechanical prostheses have a longer life, valves of this type require longterm anticoagulant treatment [11], [19].
Ionizing radiation passing through a material object, even alive, is absorbed and transfers its energy [22]. The absorbed dose is a measure of ionizing radiation absorption. The biological effects of ionizing radiation depend not only on the absorbed dose, but also on the type of radiation, the organ that has been irradiated and the type of tissue. The effect of the type of radiation depends on the dose equivalent. Each tissue or organ displays a different sensitivity to the same dose equivalent. Ionizing radiation can also affect many

* Corresponding author: Magdalena Kopernik, AGH University of Science and Technology, al. Mickiewicza 30, 30-059 Kraków, Poland. Phone: +48126175126, e-mail: kopernik@agh.edu.pl

Received: November 19th, 2019

Accepted for publication: January 19th, 2020 
processes that take place in a single cell and, therefore, the effect of radiation is largely dependent on cell's activity. This activity is determined by expression of the genes it contains, its tendency to mutate and its microenvironment, including growth factors, cytokines, nutrients surrounding the cells, and extracellular matrix to which the cells adhere. Ionizing radiation can initiate processes within the nucleus, the cell membrane, and the cytoplasm, although DNA is the most important biological target [10]. Ionizing radiation can interact directly or indirectly with DNA, inducing production of highly reactive $\mathrm{OH}^{*}$ radicals which can diffuse into the DNA and initiate its damage response. The process of cell death also can be induced by cell membrane stress, leading to oxidation and modification of lipids which serve as second messengers, switching on DNA damage-independent apoptotic pathways. Ionizing radiation can also induce premature mitosis before completion of the $\mathrm{S}$ and $\mathrm{G} 2$ phases by the cell, resulting in the so-called mitotic catastrophe which is another form of cell death associated with radiation [16].

Ionizing radiation interacts with matter by depositing energy in the disk (DNA) structure in $10^{-19}-10^{-14}$ seconds. The cell's honeycomb contains over $200 \mathrm{~cm}$ of DNA, laid in helically rolled chromatin fibers (DNA + protein). The human genome contains about 30000 genes that make up about $1 \%$ of the DNA. The remaining part of the DNA consists of non-coding sequences [4]. The critical volume of the disk when the energy reaches the critical value and the radiation losses are equal to the ionization losses is assumed to be about $10 \mathrm{~nm}$ in diameter, including the section of the DNA molecule and the distance of radial diffusion [3]. The genome is characterized by inhomogeneous radiation sensitivity [6], [5]. It is believed that only damage to certain sites in the DNA (coding sequences) can lead to lethal cell damage. The part of the genome located on the inside of the nuclear membrane within the nuclear stroma, where there are DNA strands undergoing replication and transcription, is thought to be the most sensitive. At this point, the DNA is exposed, i.e., free of histone proteins [2], [14].

Although the energy absorption in the critical volume of the biological target that leads to DNA breaks is the same in all cells, (about $30 \mathrm{eV}$ ), the biological effect depends on a series of chemical reactions that can reduce or increase the effectiveness of radiation and induce different cell activities, and thus influence the probability of the radiation interacting with the DNA molecule.

Radiation-induced changes in the cell that are unrepairable and lead to its death are called lethal conditions [21], although the cell detachment by the laser light could also influence on the key protein degradation which are responsible for the appropriate physical properties of the tissue [20]. This paper analyzes the impact of laser radiation on heart valve tissue with a numerical-mechanical interpretation of this phenomenon. Laser radiation was chosen with the aim of activating programmable cell death in the tissue to induce decellularization. The goal was not only to analyze and induce the process itself, but to develop a method that is not yet available in clinical use.

\section{Materials and methods}

The hearts of pigs were collected from adult animals weighing about $70 \mathrm{~kg}$ to $80 \mathrm{~kg}$. Experimental hearts were placed in sterile containers in Ringer's solution (Solutio Ringeri, FRESENIUS KABI) and transported on ice to the laboratory in less than 1 hour. The pulmonary and aortic valves, with the initial segment of pulmonary trunk and aorta, respectively, were removed from each heart. Samples were rinsed with Ringer's solution to remove blood and incubated for 24 hours at $4{ }^{\circ} \mathrm{C}$ in an antibiotic bath containing $1 \%$ penicillin/streptomycin (Gibco), 0.1\% Ciprinol (Krka), and $0.2 \%$ Mycomax (Zentiva) dissolved in $1000 \mathrm{ml}$ of Ringer's solution.

To remove cells from the tissue, the technique of direct exposure to laser radiation was applied. The idea of using a laser beam to remove cells from tissue is based on the assumptions of radiation therapy and the interaction of radiation with the material. All experimental studies were carried out in the setup shown schematically in Fig. 1. The laser radiation at wavelength of $1064 \mathrm{~nm}$, emitted by Q-switched Nd:YAG

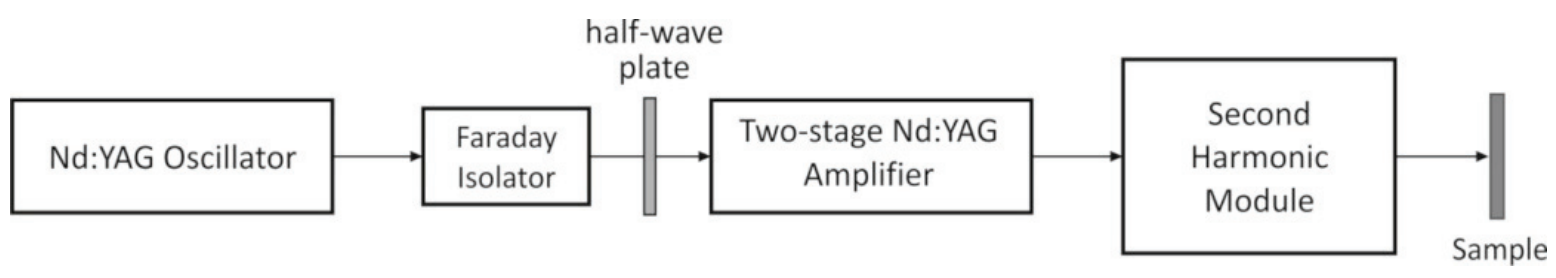

Fig. 1. Experimental setup 
oscillator was entered into two-stage laser amplifier and then, it was directed directly to the sample surface or through the second harmonic module, where it was converted into radiation at $532 \mathrm{~nm}$. The measured diameter of the laser beam incident on the tissue was about $7 \mathrm{~mm}$ for the case of fundamental radiation at $1064 \mathrm{~nm}$ and $8.5 \mathrm{~mm}$ for second harmonic at $532 \mathrm{~nm}$. It should be noted that the energy distribution in the cross-section of the beam was very closed to Gaussian pattern.

The Faraday isolator placed at the output of the oscillator was to protect the system against excitation of free generation as a result of coupling of totally reflecting mirror in the oscillator with any surface on pathway of laser radiation to the sample. Half-wave plate allowed, in turn, for the control of the polarization state of radiation on the sample.

The following parameters of the laser beam were proposed for application on the tissue (Table 1). In order to check whether there was no heat accumulation effect at repetition rate of $5 \mathrm{~Hz}$, in some cases, we performed additional tissue irradiation with the same pulse energy, but with reduced repetition rate to $1 \mathrm{~Hz}$. The obtained results were very similar in both cases, which indicated that there was no significant heat accumulation. The material, after laser irradiation, was rinsed in physiological saline and then placed in a container with $4 \%$ buffered paraformaldehyde.

Table 1. Specimen irradiation parameters

\begin{tabular}{|c|c|c|c|c|c|}
\hline $\begin{array}{l}\text { Wavelength } \\
{[\mathrm{nm}]}\end{array}$ & $\begin{array}{c}\text { Beam } \\
\text { diameter } \\
{[\mathrm{mm}]}\end{array}$ & $\begin{array}{c}\text { Pulse } \\
\text { energy } \\
{[\mathrm{mJ}]}\end{array}$ & $\begin{array}{l}\text { Energy } \\
\text { density } \\
{\left[\mathrm{J} \cdot \mathrm{cm}^{-2}\right]}\end{array}$ & $\begin{array}{c}\text { Repetition } \\
\text { rate } \\
{[\mathrm{Hz}]}\end{array}$ & $\begin{array}{c}\text { No. } \\
\text { of } \\
\text { pulses }\end{array}$ \\
\hline \multirow{13}{*}{1064} & \multirow{13}{*}{7} & \multirow{6}{*}{65} & \multirow{6}{*}{0.169} & 1 & 100 \\
\hline & & & & 5 & 10 \\
\hline & & & & 5 & 50 \\
\hline & & & & 5 & 100 \\
\hline & & & & 5 & 200 \\
\hline & & & & 5 & 500 \\
\hline & & \multirow{7}{*}{32} & \multirow{7}{*}{0.083} & 5 & 10 \\
\hline & & & & 5 & 50 \\
\hline & & & & 1 & 100 \\
\hline & & & & 5 & 100 \\
\hline & & & & 1 & 200 \\
\hline & & & & 5 & 200 \\
\hline & & & & 5 & 500 \\
\hline \multirow{11}{*}{532} & \multirow{11}{*}{8.5} & \multirow{5}{*}{62} & \multirow{5}{*}{0.109} & 1 & 100 \\
\hline & & & & 5 & 10 \\
\hline & & & & 5 & 50 \\
\hline & & & & 5 & 100 \\
\hline & & & & 5 & 200 \\
\hline & & \multirow{6}{*}{32} & \multirow{6}{*}{0.056} & 5 & 500 \\
\hline & & & & 5 & 200 \\
\hline & & & & 1 & 100 \\
\hline & & & & 5 & 100 \\
\hline & & & & 5 & 10 \\
\hline & & & & 5 & 50 \\
\hline
\end{tabular}

The decellularization process can be induced by chemical and physical factors. The physical factor can be an acoustic wave or laser light. There are some examples of the use of both physical factors in medicine [8], [18], as well as examples of their simulations, and the application of acoustic waves in medicine using the FEM (finite element method) [18]. In the case of FEM models, in which the factor that loads the material is a wave, the size of the finite element and the length of the time step should be properly matched and appropriately selected by analyzing the wavelength and its period.

The purpose of modeling as a part of this paper was to investigate the mechanical effects of wave loading on the heart valve tissue material. Thus, a numerical model of the experiment was developed, mapping its main components and mechanical conditions. In the experiment, the sample was irradiated with laser pulses of $32 \mathrm{~mJ}$ or $62 \mathrm{~mJ}$ in energy for the beam diameter of $8.5 \mathrm{~mm}$ in the case of second harmonic, and $32 \mathrm{~mJ}$ or $65 \mathrm{~mJ}$ for the diameter equal to $7 \mathrm{~mm}$ in the case of fundamental radiation (Table 1). In the simulation, a case with maximum energy density, for the highest pulse energy of $65 \mathrm{~mJ}$ and beam diameter of $7 \mathrm{~mm}$, was used for sample loading. The mechanical effect of laser radiation interaction with the material of the heart valve tissue is the maximum for these sample loading conditions and this was confirmed in the test simulations. The radial energy density variation of the laser beam is described by the Gaussian function:

$$
I(r)=\frac{2 E}{\pi w^{2}} \exp \left[\frac{-2 r^{2}}{w^{2}}\right],
$$

where $E$ is the pulse energy contained in the entire beam, $w$ is the spot size and $r$ is the beam radius.

Therefore, the sample loading in the simulation is as per the function described in Eq. (1) in which the energy density depends exponentially on the radius of the beam.

The sample in the experiment lies motionless on the substrate, and the contact between the sample and the substrate can be determined by adhesive forces. The contact between the sample and the substrate was determined using a friction model, and the friction coefficient was assumed to be 0.1 in the simulation. The substrate on which the sample lies is immobilized from the bottom in the simulation, while the edges of the sample remain free. The models of the sample and substrate also take into account the load of the system by the force of gravity. Thus, the boundary conditions used in the simulation correspond exactly to the experimental conditions. 
The 3D FEM model of the sample and substrate, in which the sample is loaded by the mechanical wave at its center point from the top (focus of the wave), was developed in the Abaqus 6.14-5 software and 8-node, linear brick, FE were used to build the model. As in the experiment, a rectangular sample with dimensions of $20 \times 20 \times 0.5 \mathrm{~mm}$ was developed. The dimensions of the substrate in the model can be defined as infinite in each direction. However, the simulation includes a finite solid model of the substrate but as it significantly exceeds the sample size, the above assumption is still met.

In the simulation, FEM models of the samples were generated for the materials of the pulmonary and aortic valve tissues, whose mechanical properties were identified on the basis of the uniaxial tensile test results [21]. The tensile test results show the elastic and plastic properties of the heart tissues; the elastic range is very wide and the plastic range is very narrow. The determined elastic properties of tissues are: Young's modulus equal to $11.68 \mathrm{MPa}$ for the pulmonary valve and 17.95 $\mathrm{MPa}$ for the aortic valve, and Poisson's ratio equal to 0.5 for both models of valve tissue samples. The elastic properties of the substrate material are: Young's modulus of $300 \mathrm{GPa}$ and Poisson's ratio of 0.3. The same type of FE mesh elements are generated for the substrate and the sample, and the FE meshes formed have similar density.

Two simulations were performed, one for the aortic valve sample model and one for the pulmonary valve sample model, using maximum load. Explicit dynamic analysis was applied in the simulations [13]. Each simulation required about 200 iterations. The average computing time for each simulation was about five hours using multiple processors on a standard PC.

The efficiency of cell debridement was verified by immunofluorescence staining involving the staining of cell nuclei. The cell nuclei, after permeabilization, were labeled with a 4', 6-diamidino-2-phenylindole dye, an organic, aromatic, heterocyclic compound, an amine that binds to DNA, and a highly fluorescent dye. The tests were performed using Exciter 5, a confocal laser scanning microscope.

The effect of the laser beam on tissue, and in particular on changing the amount of genetic material, was analyzed on a molecular scale using quantitative polymerase chain reaction (qPCR) analysis. Small (10-200 ng) amounts of genomic DNA are needed for the study and the population of analyzed particles is very homogeneous. Experimental formaldehyde-fixed specimens of aortic valvular rings and semilunar valves (approx. $100 \mathrm{mg}$ ) were weighted and minced using a disposable surgical blade and added 10 pg PGEM plasmid (Applied Biosystems, Foster City, CA) as a internal standard. Total cellular DNA was extracted using a commercial kit (Sherlock AX, A\&A Biotechnology, Gdynia, Poland), which is based on proteinase $\mathrm{K}$ digestion and ion-exchange minicolumn purification. The eluent was precipitated and re-dissolved in $30 \mu \mathrm{l}$ TRIS-EDTA buffer $10 / 1 \mathrm{mM}$. Amplification of porcine (Sus scrofa domestica) mitochondrial 16S rRNA gene was done using primers: 5'-GCC TGT TTA CCA AAA ACA TCA (forward) and 5'-CTC CAT AGG GTC TTC TCG TCT T (reverse) resulting in 243 base pairs product. A hot-start commercial Sybr Green PCR Master Mix was used for real-time amplification in 7900 HT thermal cycler (Applied Biosystems). Reaction was conducted in $20 \mu \mathrm{l}$ volume using $4 \mu \mathrm{l}$ of extracted DNA. All samples were measured simultaneously in a single 96-well plate in duplicates. Results were presented as cycle threshold denoting the thermal cycle, at which tracer dye exceeded arbitrary fluorescence level, corrected for DNA extraction efficiency by a cycle treshold of PGEM amplification using a conventional M13 primer pair. This value shows an excellent negative correlation with a logarithm of initial concentration of the template DNA.

The samples for microscopic examination, following standard histological techniques, were fixed for 24 hours in $4 \%$ buffered paraformaldehyde, dehydrated with increasing concentrations of alcohol, embedded with paraffin and cut into $6 \mu \mathrm{m}$ thick sections using a microtome. Deparaffinized sections were then stained with hematoxylin and eosin to determine the preservation of the general histological structure and to evaluate the efficiency of cell debridement. Additionally, staining was used to assess preservation of the vascular elastic elements.

The specimens were examined under an Olympus IX83 light microscope. Images were collected and morphometric analysis was carried out using the DP-73 digital CCD camera (Olympus, Japan) and the CellSens Dimension (Olympus, Japan) image analysis system.

\section{Discussion}

In the presentation of results obtained from the FE models of wave-loaded aortic and pulmonary samples and substrates, the results calculated for the FE model of substrates were not considered, as the experiment also did not examine the impact of the load on the substrate material. The FE model of the substrate was introduced to correctly represent the boundary conditions for the FE models of the tissue 
(a)

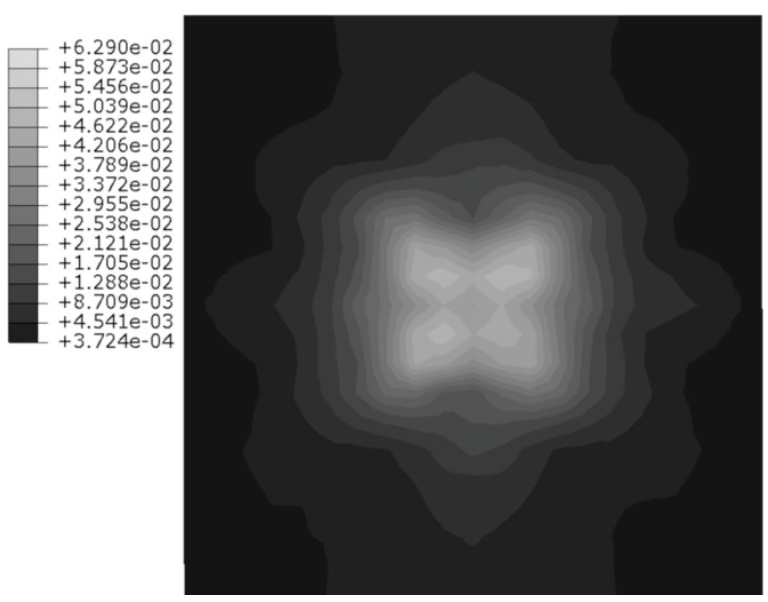

(b)

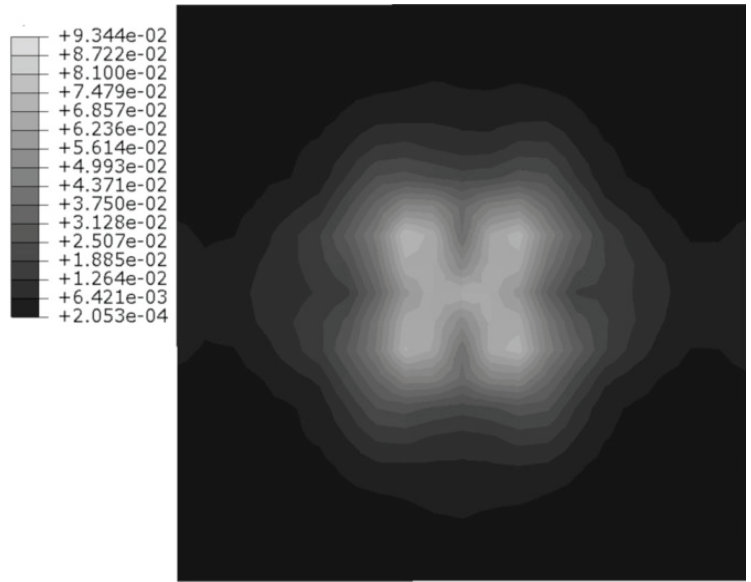

Fig. 2. Distributions of von Mises stress (MPa) for: (a) aortic, b) pulmonary valve models (top view)
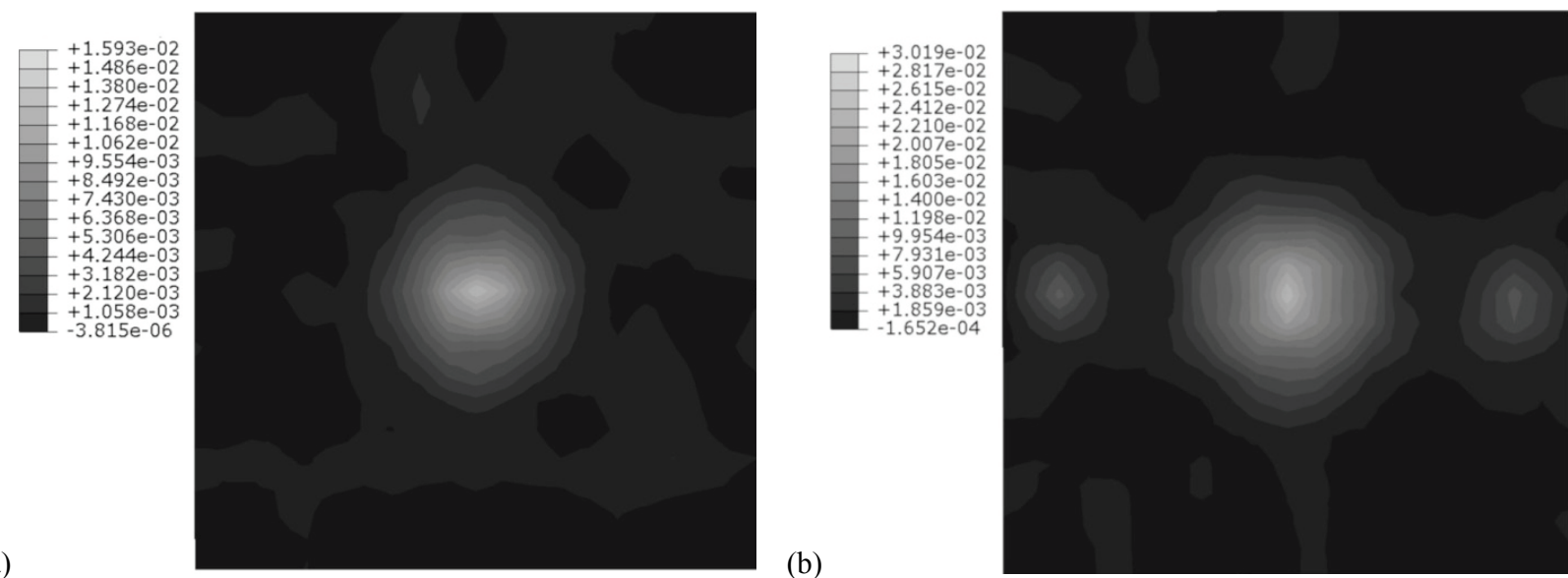

Fig. 3. Distributions of maximum principal strain for: (a) aortic, (b) pulmonary valve models (top view)
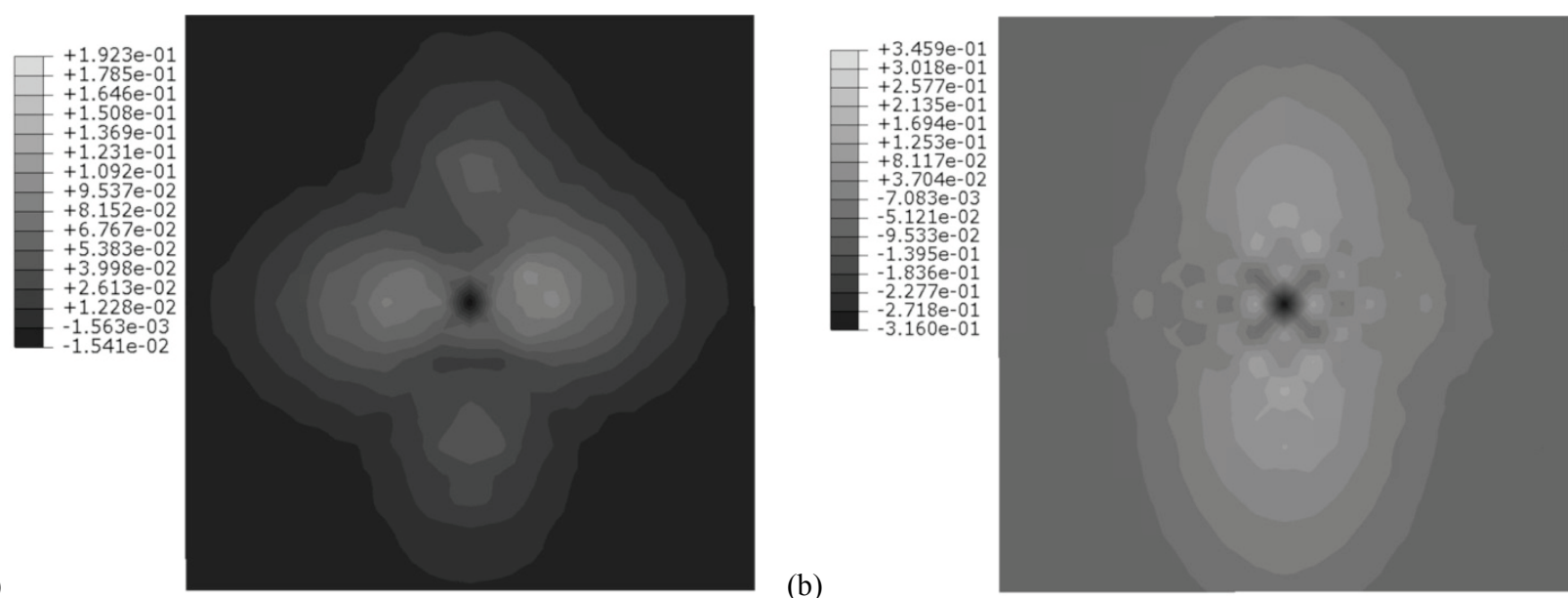

Fig. 4. Distributions of $Z$-displacement (mm) for: (a) aortic, (b) pulmonary valve models (top view)

samples. The biomechanical properties of aortic and pulmonary valve tissues can be modified by the process of decellularization and are shown in the present section. The decellularization process of the heart valve tissue also results in changes in the hydrodynamic properties of the valves which are not discussed in this paper but shown for example purposes in literature [7]. 
The simulation results are obtained for two models of tissue samples loaded by the same distribution of maximum energy density and gravity. The results are presented as distributions of von Mises stress, maximum principal strain and displacement in the $Z$ direction which is the direction of wave-loading and gravity (Figs. 2-4) in the last calculation step of each simulation on the surface of the sample model.

For the aortic valve model in the last calculation step of the simulation, the maximum von Mises stress of $62.9 \mathrm{kPa}$ occurs at the center of the model and has several maxima in an area of $4 \times 4 \mathrm{~mm}$ (Fig. 2a). However, the maximum principal strain has one maximum in an area of $1 \times 1 \mathrm{~mm}$ at the center of model and it is equal to 0.0159 (Fig. 3a). The displacement in the $Z$ direction has one minimum at the center of the model equal to $0.0154 \mathrm{~mm}$ in an area of $1 \times 1 \mathrm{~mm}$, and has several maxima in an area of $6 \times$ $6 \mathrm{~mm}$ which surrounds the minimum (Fig. $4 \mathrm{a}$ ).

For the pulmonary valve model in the last calculation step of the simulation, the maximum von Mises stress of $93.44 \mathrm{kPa}$ occurs at the center of the model and has several maxima in an area of $4 \times 4 \mathrm{~mm}$ (Fig. 2b). The maximum principal strain has one main maximum in an area of $1 \times 1 \mathrm{~mm}$ at the center of model and it is equal to 0.03 (Fig. 3b). There are also two local minima on the main axis of the sample (along the $X$ direction) near the edge of the sample. The displacement in the $Z$ direction has one minimum at the center of the model equal to $0.31 \mathrm{~mm}$ in an area of $1 \times 1 \mathrm{~mm}$, and has several maxima in an area of $3 \mathrm{~mm}$ $\times 6 \mathrm{~mm}$ which surrounds the minimum (Fig. $4 \mathrm{~b}$ ).

In Figure 5, the change in $Z$ displacement with distance from the center of each sample in the last computational step for both valves is shown. The dis- tance is measured from the center of the sample to its right edge along a straight line, and the data is collected from the nodes of FE elements on this straight line. The graph shown in Fig. 5 corresponds to the data shown in Fig. 4, in which the $Z$ displacement distribution is shown for the entire surface of the samples for both valves.

A comparison of computed strains, stresses and $Z$-displacements at the center of the models shows that the biggest differences occur between the values of strains and displacements for both models, whereas the values of stresses observed in the models differ by the smallest percentage. The largest values of von Mises stress, maximum principal strain and displacements are computed for the pulmonary valve tissue which is a less elastic material than the aortic valve tissue. The mechanical properties of both tissues are presented in Chapter 2 along with simulation parameters.

As a result of wave influence on the surface of the model, a cavity is formed and the wave propagates over the entire surface. The experimental observations of the effects of the wave on the surface of the samples are analogous, and the dimensions of the cavity are also same.

The maximum stresses obtained in the model after the application of a load in the form of a wave of a given amplitude were $0.063 \mathrm{MPa}$ in the aortic valve material and $0.093 \mathrm{MPa}$ in the pulmonary valve material. In the literature, the highest ultimate tensile strength of the aorta is reported to be in the range of 0.3-0.8 MPa [12]. However, stretched elastin fiber taken from the aorta has the highest tensile strength of 1.5 MPa. In the literature, elastin properties have also been studied as a function of the preparation and stor-

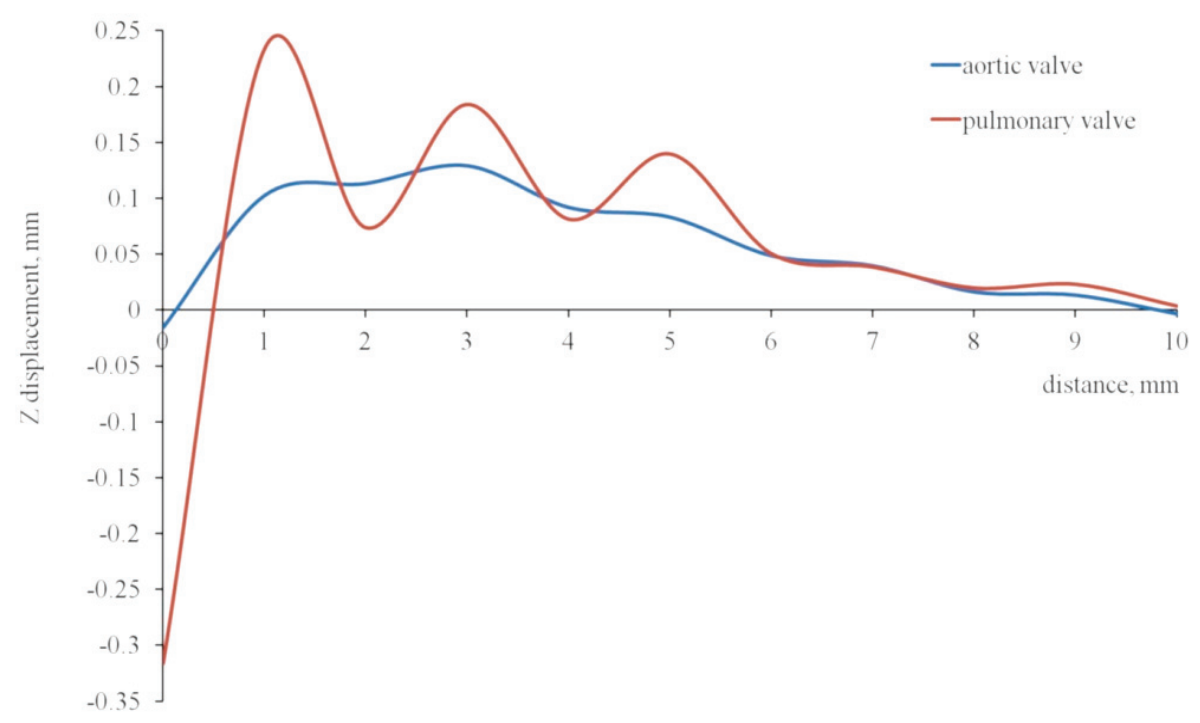

Fig. 5. Z-displacement versus distance from the center of the specimen for the tissue of aortic and pulmonary valves 
age conditions of elastin fibers [20], [15]. This approach results in providing a wider range of critical stresses which can range from 0.2 to $2.9 \mathrm{MPa}$ [15], [17]. Thus, the values obtained at the modeling stage in this work are not critical for elastin fibers, compared with literature data. It should be noted that, in the literature, the fibers are subjected to tension, while in the present work the entire sample is subjected to compression and the models do not take into account the anisotropy of mechanical properties.

The results of the numerical analysis of the mechanical effect of the wave causing decellularization

(a)
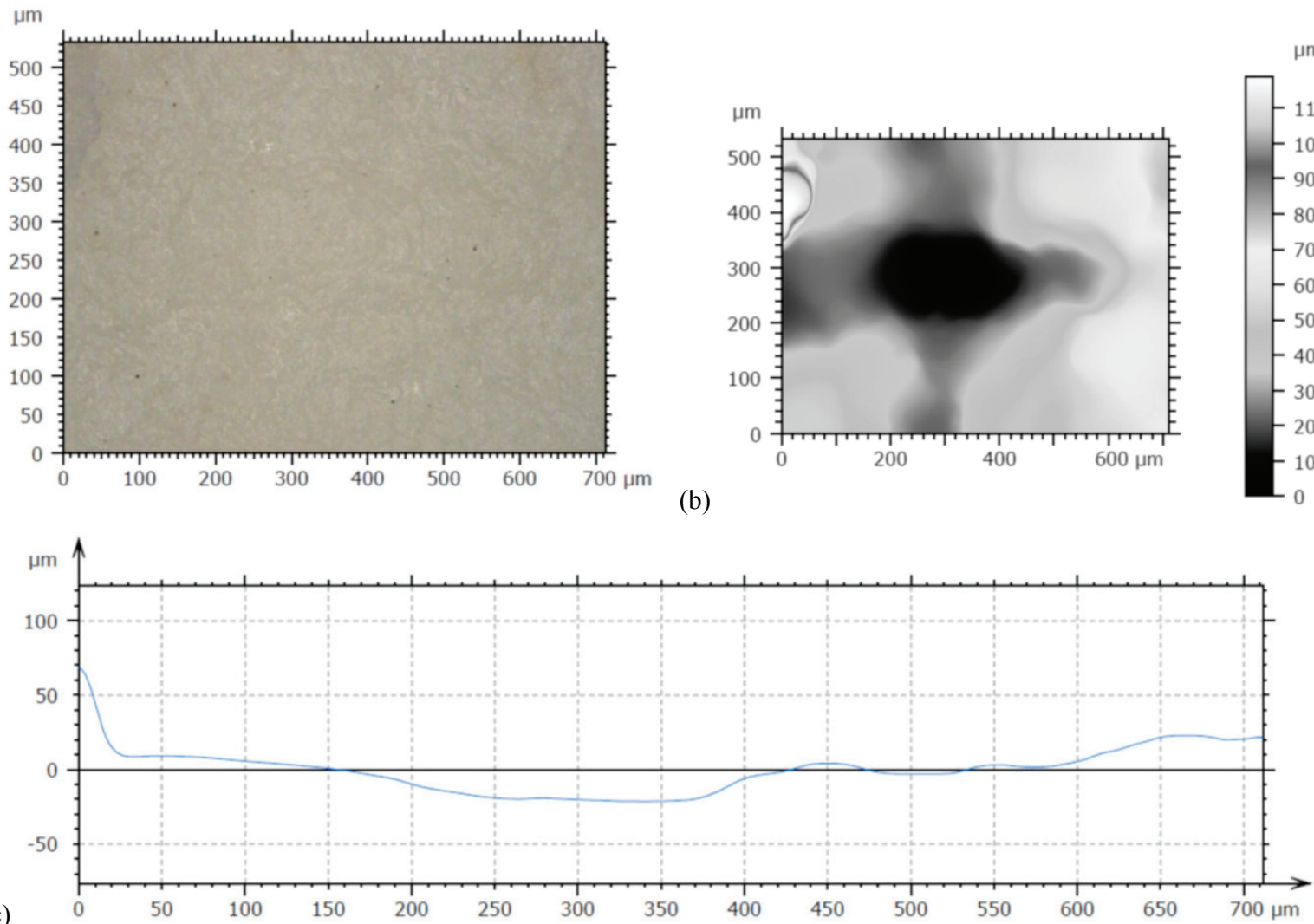

Fig. 6. Topography $[\mu \mathrm{m}]$ of aortic valve models for: (a) surface, (b) height map, (c) profile
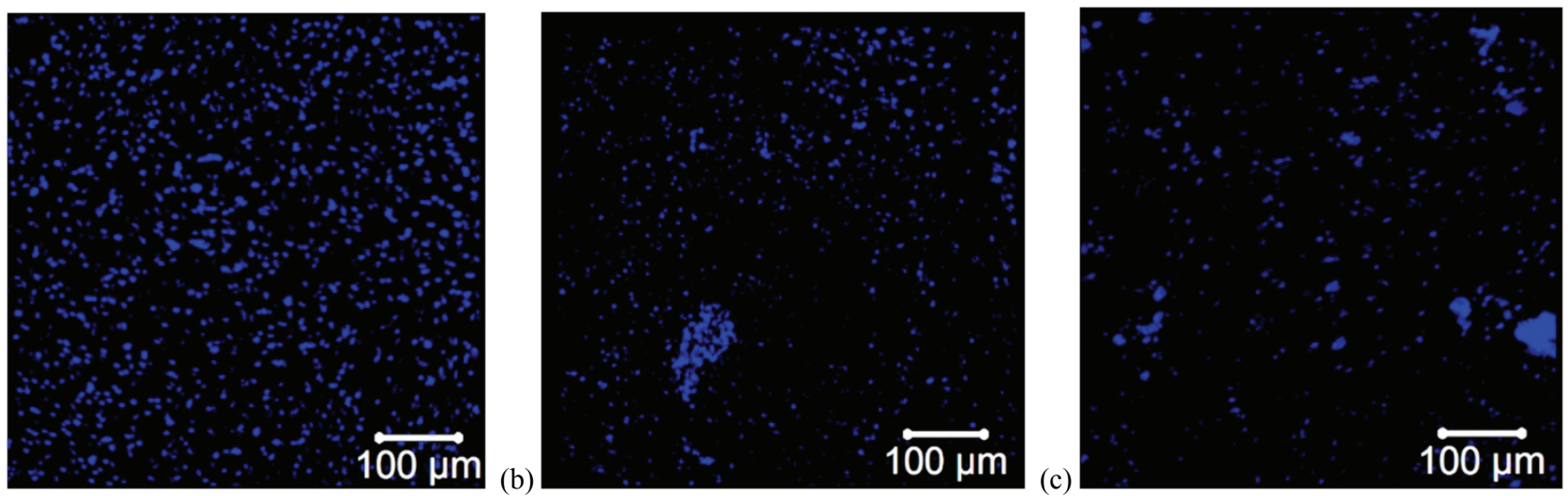

Fig. 7. Distributions of the 6-diamidino-2-phenylindole dye (DAPI)-stained nuclei analyzed by confocal microscopy for:

(a) native tissue, (b) 200 pulses, (c) 500 pulses areas of the tissue which interacted with the laser beam was examined with a digital microscope (Fig. 6). Figure $6 \mathrm{a}$ represents the surface of the tested tissue - snap shot in $X Y$ plane. Figure $6 \mathrm{~b}$ represents the surface of the tested tissue - height map in $X Y$ plane and colored $Z$ scale indication. Fig. 6c shows the surface of the tested tissue - profile in $X Z$ direction. The strongest effect was observed at $532 \mathrm{~nm}$ wavedeflections in line with predictions obtained by numerical analysis. were verified experimentally. The topography of the length and $32 \mathrm{~mJ}$ of energy. Tissue tests showed

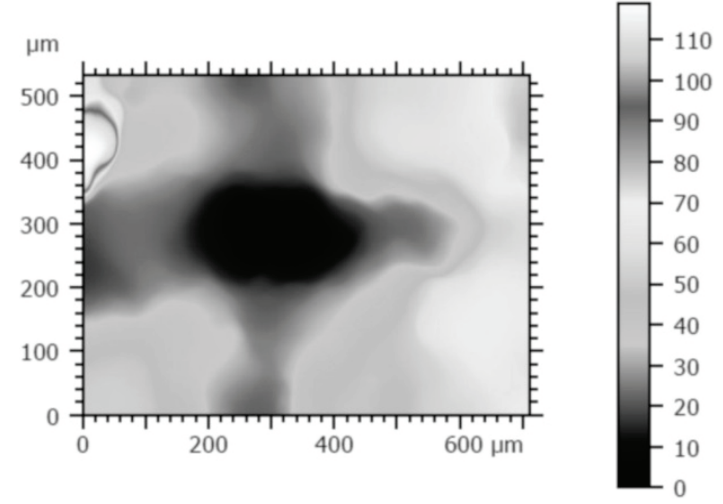


Investigation of the number of nuclei remaining in the tissue searched for the tissue in which the strongest deflection effect, resulting from interaction with the laser beam, was observed. Fluorescence analyses were carried out for tissue exposure to 200 and 500 laser pulses. The results of the tests are shown in Fig. 7.

Analysis of the optical cross-section of the tissue allowed the estimation of possible voids associated with the elimination of cell nuclei from the tissue (Fig. 8). As shown by cross-sectional analysis, voids were found at the cumulative locations of the interfering laser beam. The visible effect of eliminating cells from tissue is not uniform. The heterogeneity of the distribution of the caves on the surface of the samples was also confirmed in the simulation by the alternate occurrence of local minima and maxima of strain and stress. The latter observation also results from the uneven removal of cells from the surface of the samples as was proved earlier.

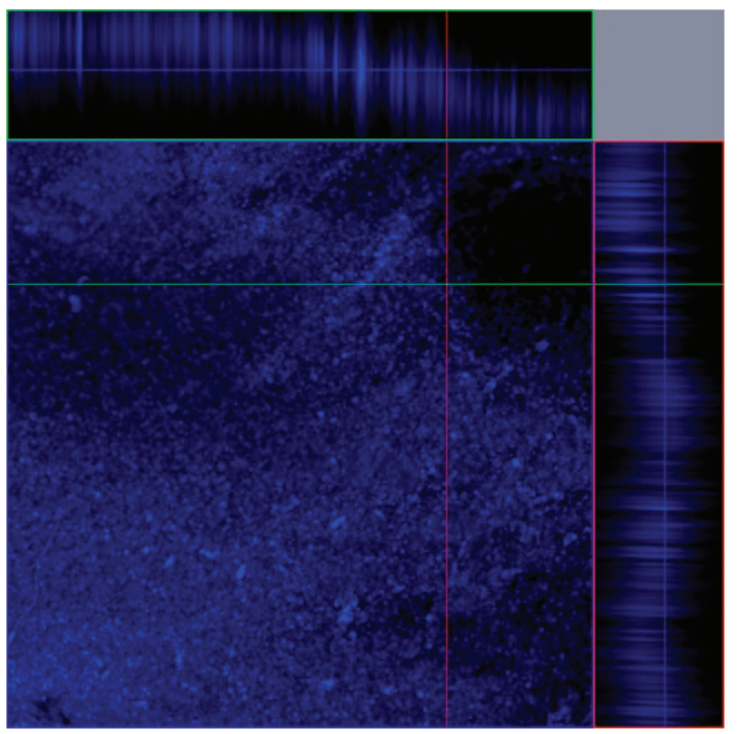

Fig. 8. Optical immunofluorescent $X-Y$ cross-section of the DAPI-stained decellularized tissue

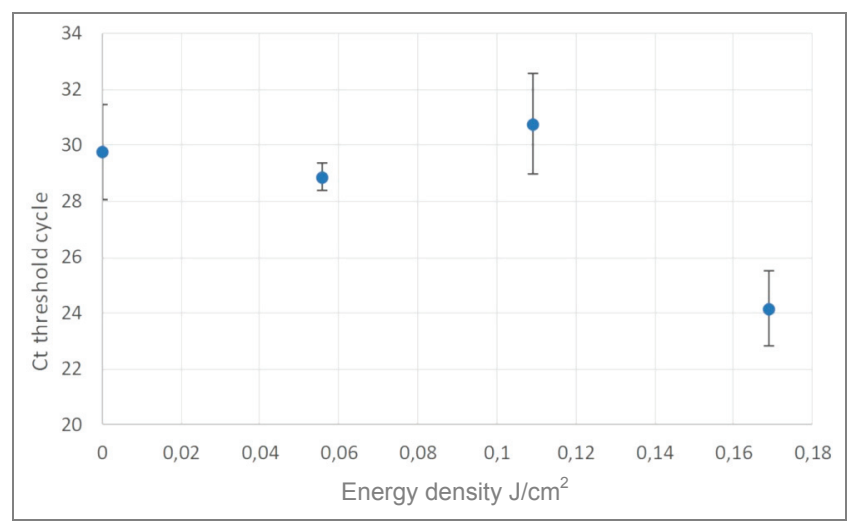

Fig. 9. Energy density influence on the threshold cycle
The influence of energy density on change threshold cycle $C_{t}$ was tested. The results are presented in Fig. 9. The reduction of energy does not affect the reduction of the amount of genetic material in the examined tissue sample. The reduction in DNA is visible for high energy densities. Selected laser parameters were used to analyze the impact of the number of pulses on the amount of genetic material (Fig. 10).

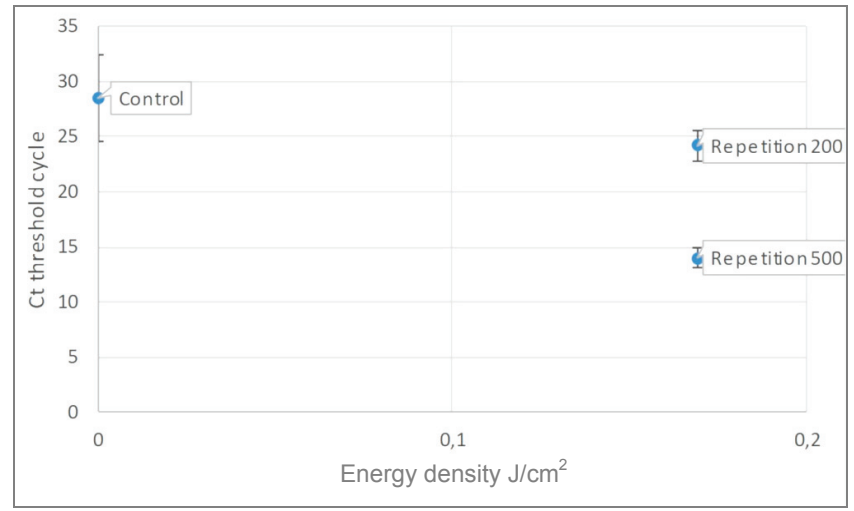

Fig. 10. Energy density and the repetition influence on the threshold cycle

Total cell removal was not achieved in any irradiated material. The persistent cells showed signs of subtle, moderate or significant damage, as indicated by their substantial deformations and/or cytoplasmic condensations, as well as deformation or pycnotization of their nuclei. When compared with the native tissue (Fig. 11), the irradiated material revealed some changes in the organization of the extracellular substance including stromal edema, reduction in collagen and elastic fiber crosslinks with disorganization and/or fragmentation of fibrous elements. These changes had different degrees of severity and spread (Figs. 12 and 13), from small, focal lesions to significant disorganization occupying a large area of the arterial media. In some cases, particularly in pulmonary artery samples, significant disintegration and weakening of the stroma resulted in the occurrence of focal, single or multiple stromal tears which could have been formed during the sectioning of the tissue material (Fig. 13).

The goal of this work was to create a fully functional tissue. Aortic valves of animal origin were utilized in this study. The host cells were removed (decellularized) by physical means using laser light irradiation. The mechanical results represented by displacement at the center of the sample were coherent and the heterogeneity of the distribution of the caves on the surface of the samples was confirmed, both by experiment and in the simulation by the alternate occurrence of local minima and maxima. The latter results from the 
(a)
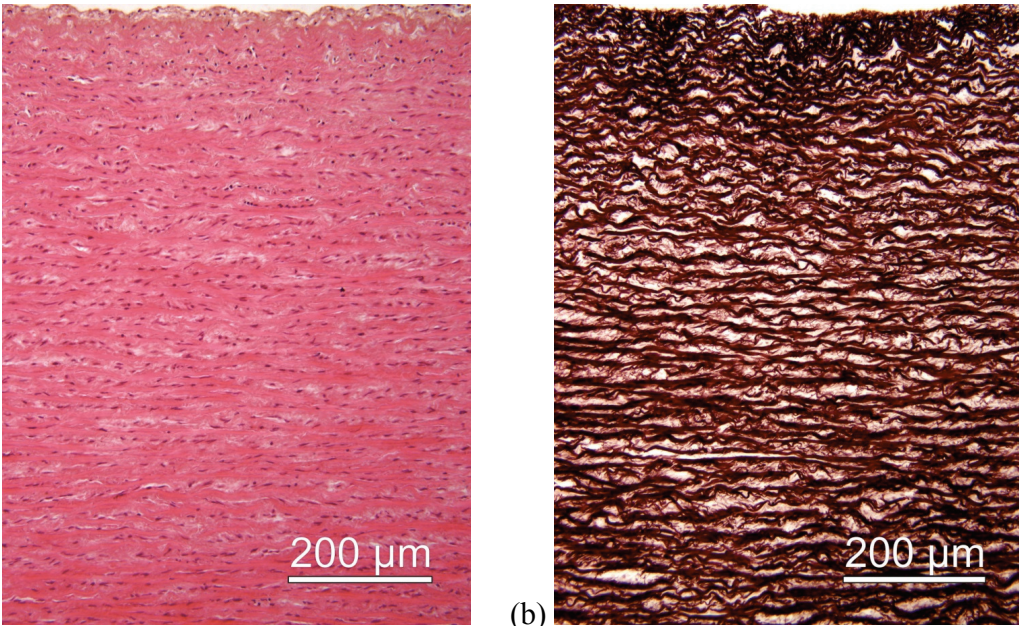

Fig. 11. Micrographs of the wall of native aorta showing: (a) regular distribution and parallel alignment of cells (HE staining), (b) wavy course and parallel arrangement of elastic lamellae with thinner elastic fibers between them (orcein staining)

(a)

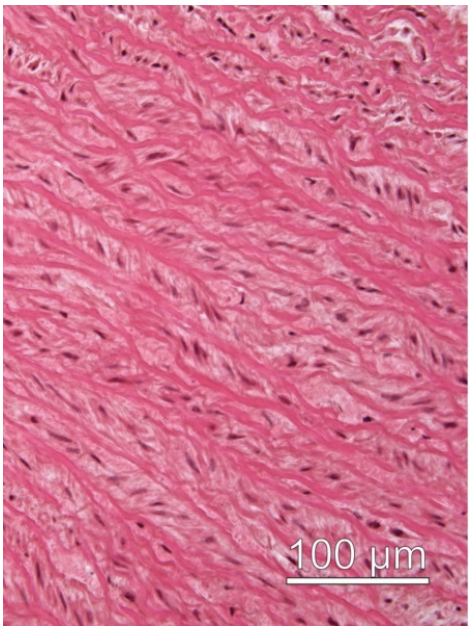

(b)

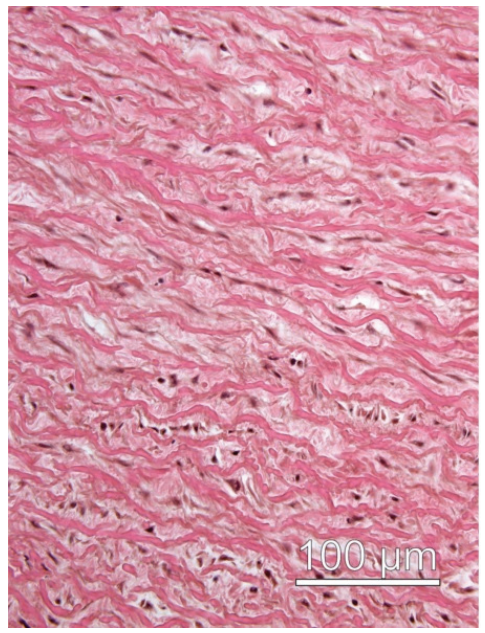

(c)

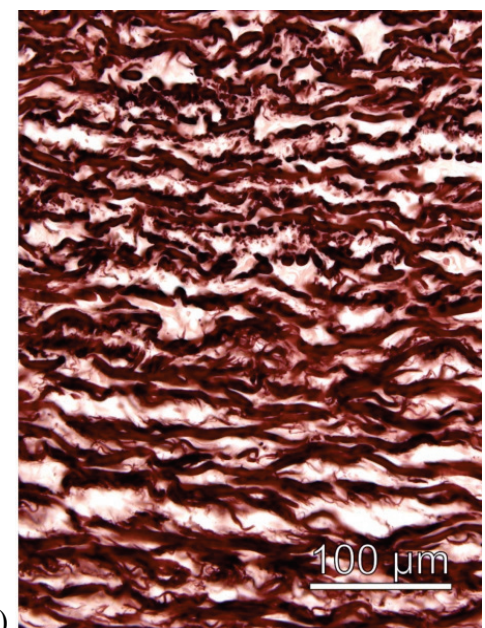

Fig. 12. Micrographs of the aortic walls subjected to ablation procedure demonstrating:

(a) minor stromal edema and cell deformation, (b) significant stromal injury with partial cell loss,

(c) reduction of crosslinks and partial fragmentation of elastic elements stained with orcein

(a)

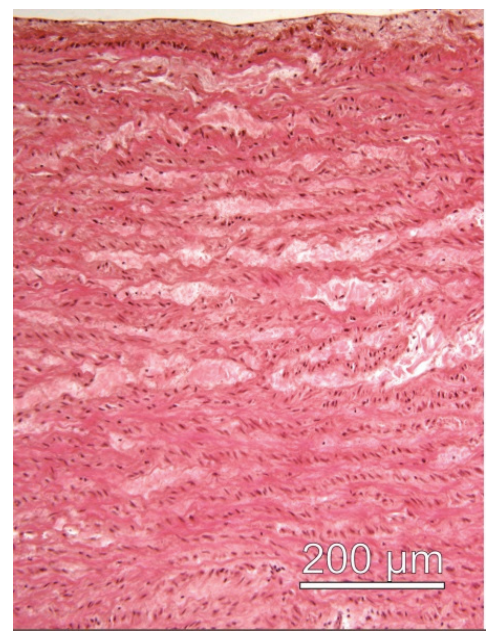

(b)

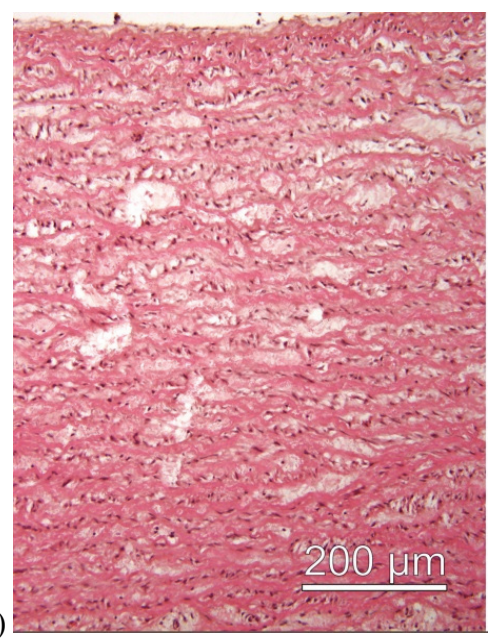

(c)

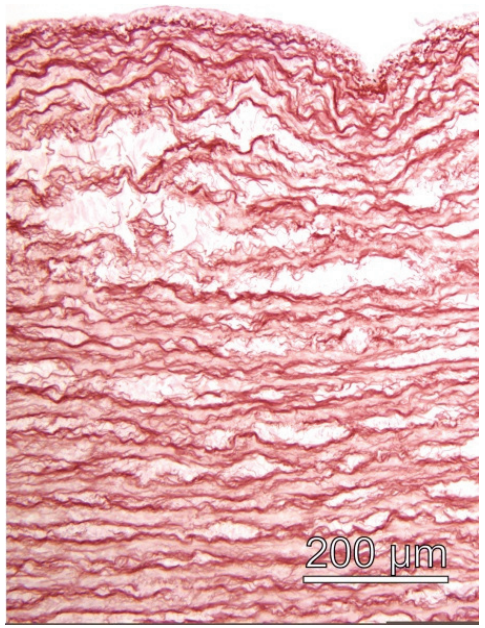

Fig. 13. Micrographs of the pulmonary artery walls subjected to ablation with different degrees of stroma injury:

(a) swelling of the more superficial part of the media with partial disorganization of both cellular and non-cellular elements,

(b) significant structural disorganization seen in the whole thickness of the wall,

(c) focal stromal tears and fragmentation of elastic elements stained with orcein 
uneven removal of cells from the effect of the wave causing decellularization were also predicted by the numerical model. The purpose was to eliminate cells without degradation of the extracellular matrix. Generation of the controlled process by induced mutations in the cell's DNA leads to the decellularization process. It should have triggered the activation of proteins responsible for controlling the correctness of the genetic code and directed the cells to the programmable death pathway. During the cell cycle, the checkpoint protein cascade activation should lead to eliminating defective cells by directing them to the programmable death pathway. These methods differ in their effectiveness from the conventional chemical method. Laser radiation had a destructive effect on the components of the extracellular matrix (e.g., collagen and elastic fibers), mainly depending on the fluence and number of pulses in a single exposure. Thus, the decellularization process was controlled by histological methods. It should be emphasized that due to low absorption and high scattering, laser radiation can deeply penetrate the tissue, which allows for effective decellularization process in the entire volume of irradiated tissue.

It was found that physical methods (laser radiation) were an alternative to chemical methods. Physical methods do not dissolve the tissue in case of longterm depigmentation. For certain laser radiation parameters, the matrix fibers were destroyed. Choosing the right pulse energy, repetition rate and wavelength will eliminate this. Chemical techniques are effective and refined. It is not possible to carry out the process of chemical accreditation without a compromise related to the thickness of the tissue. These methods work well for tissues with homogeneous thicknesses. In the case of surrogates, where the tissue has a heterogeneous thickness, it is necessary to use other alternative techniques.

\section{Conclusions}

Physical methods can be an alternative to chemical methods to create a fully functional tissue. The host cells were removed by physical means using laser light irradiation. The purpose was to eliminate cells without degradation of the extracellular matrix. Generation of the controlled process by induced mutations in the cells' DNA leads to the decellularization process.

Laser radiation had a destructive effect on the components of the extracellular matrix (e.g., collagen and elastic fibers), mainly depending on the fluence and number of pulses in a single exposure. The differences between the valve tissue materials were shown, and the impact of the process of decellularization on the properties of the tissues analyzed. Thus, the decellularization process was controlled by histological methods.

Due to low absorption and high scattering, laser radiation can deeply penetrate the tissue, which allows for effective decellularization process in the entire volume of irradiated tissue.

The mechanical results represented by displacement at the center of the sample were coherent and the heterogeneity of the distribution of the caves on the surface of the samples was confirmed, both by experiment and in the simulation by the alternate occurrence of local minima and maxima. The latter results from the uneven removal of cells from the effect of the wave causing decellularization were also predicted by the numerical model.

\section{Acknowledgements}

The research was partially financed by the Polish National Science Centre under Project No. UMO-2016/23/B/ST8/01481 "Interdisciplinary methods of creating and functioning of biomimetic materials based on Animal origin extracellular matrix". Part of the work was executed under the project sponsored by the European Union from resources of the European Social Fund (Project No. WND-POWR.03.02.00-00-I043/16).

\section{References}

[1] ArZani A., Mofrad M.R.K., A strain-based finite element model for calcification progression in aortic valves, J. Biomech., 2017, 65, 216-220.

[2] BedfFord J.S., Phil D., Sublethal damage, potentially lethal damage, and chromosomal aberrations in mammalian cells exposed to ionizing radiations, Int. J. Radiat. Oncol. Biol. Phys., 1991, 21, 1457-1469.

[3] BoHR V.A., DNA repair at the level of the gene: molecular and clinical considerations, J. Cancer Res. Clin., 1990, 116, 384-391.

[4] Boothman D.A., Bouvard I., Hughes E.N., Identification and characterization of X-ray-induced proteins in human cells, Cancer Res., 1989, 49, 2871-2878.

[5] Cregan S.P., Smith B.P., Brown D.L., Mitchel R.E.J., Two pathways for the introduction of apoptosis in human lymphocytes, Int. J. Radiat. Biol., 1999, 75, 1069-1086.

[6] CURTIS S., Lethal and potentially lethal lesions induced by radiation - An unifield repair model, Radiat. Res., 1986, 106, 252-270.

[7] Desai A., Vafaee T., Rooney P., Kearney J.N., Berry H.E., INGHAM E., FISHER J., JENNINGS L.M., In vitro biomechanical and hydrodynamic characterisation of decellularised human pulmonary and aortic roots, J. Mech. Behav. Biomed., 2018, $79,53-63$. 
[8] Douglas T.E.L., KroK-Borkowicz M., Macuda A., Pietryga K., PAMUŁA E., Enrichment of thermosensitive chitosan hydrogels with glycerol and alkaline phosphatase for bone tissue engineering applications, Acta Bioeng. Biomech., 2016, 18, 51-57.

[9] Filova E., Straka F., Mirejovsky T., Masin J., Bacakova L., Tissue-engineered heart valves, Physiol. Res., 2009, 58, 141-158.

[10] Fuller N., Lerebours A., Smith J.T., Ford A.T., The biological effects of ionising radiation on Crustaceans: A review, Aquat. Toxicol., 2015, 167, 55-67.

[11] HeAD S.J., CEliK M., KaPPETEIN A.P., Mechanical versus bioprosthetic aortic valve replacement, Eur. Heart J., 2017, $38,2183$.

[12] Holzapfel G.A., Gasser T.C., Stadler M., A structural model for the viscoelastic behavior of arterial walls: Continuum formulation and finite element analysis, Eur. J. Mech. A - Solid, 2002, 21, 441-463.

[13] KOPERNIK M., Shape optimisation of a ventricular assist device using a VADFEM computer program, Acta Bioeng. Biomech., 2013, 15, 81-87.

[14] Kugel C., Bailly I., Tourdes F., Poncy J.-L., In vitro radiation-induced effects on rat tracheal epithelial cells: i) different radiosensitivity of cell inactivation after $\alpha$ and $\gamma$ irradiations, J. Radiat. Res., 2002, 43, 27-34.

[15] Lillie M.A., GosLine J.M., The viscoelastic basis for the tensile strength of elastin, Int. J. Biol. Macromol., 2002, 30, 119-127.
[16] Maier P., Hartmann L., Wenz F., Herskind C., Cellular pathways in response to ionizing radiation and their targetability for tumor radiosensitization, Int. J. Mol. Sci., 2016, 17, 1-32.

[17] MuiznieKs L.D., KeELey F.W., Molecular assembly and mechanical properties of the extracellular matrix: A fibrous protein perspective, BBA - Mol. Basis Dis., 2013, 1832, 866-875.

[18] Pereira D., Haiat G., Fernandes J., Belanger P., Simulation of acoustic guided wave propagation in cortical bone using a semianalytical finite element method, J. Acoust. Soc. Am., 2017, 141, 2538-2547.

[19] Poli D., Antonucci E., Pengo V., Migliaccio L., Testa S., Lodigiani C., Coffetti N., FAcchinetti R., Serricchio G., Falco P., Mangione C., Masottini S., Ruocco L., De CAterina R., Palareti G., Mechanical prosthetic heart valves: Quality of anticoagulation and thromboembolic risk. The observational multicenter PLECTRUM study, Int. J. Cardiol., 2018, 267, 68-73.

[20] SkopinsKa-WiSniewsKa J., SionKowsKa A., KaminSKa A., KAZNICA A., JACHIMIAK R., DREWA T., Surface characterization of collagen/elastin-based biomaterials for tissue regeneration, Appl. Surf. Sci., 2009, 255, 8286-8292.

[21] WilczeK P., GaCh P., Jendryczko K., Marcisz M., Wilczek G., Major R., Mzyk A., Sypien A., SAmotus A., Biomechanical and morphological stability of acellular scaffolds for tissue-engineered heart valves depends on different storage, J. Mater. Sci.: Mat. Med., 2018, 29, 106-122.

[22] ZAKARIYA N.I., Benefits and biological effects wof ionizing radiation, Sch. Acad. J. Biosci., 2014, 2, 583-591. 